\title{
Philosophiques
}

\section{Après Rawls : la notion de raisonnable comme outil critique}

\section{Kai Nielsen}

Volume 24, numéro 1, printemps 1997

Avez-vous lu Rawls?

URI : https://id.erudit.org/iderudit/027421ar

DOI : https://doi.org/10.7202/027421ar

Aller au sommaire du numéro

Éditeur(s)

Société de philosophie du Québec

ISSN

0316-2923 (imprimé)

1492-1391 (numérique)

Découvrir la revue

Citer cet article

Nielsen, K. (1997). Après Rawls : la notion de raisonnable comme outil critique. Philosophiques, 24(1), 9-23. https://doi.org/10.7202/027421ar

\section{Résumé de l'article}

La notion de raisonnable est omniprésente dans l'oeuvre de Rawls. Pourtant, plusieurs estiment que cette notion est problématique et qu'elle ne saurait avoir la portée critique dont Rawls l'investit. Dans cet article, je me propose de clarifier cette notion en soutenant que dans certaines de ses articulations conceptuelles, la notion de raisonnable peut effectivement jouer le rôle critique que lui assigne Rawls. 


\title{
APRÈS RAWLS : IA NOTION DE RAISONNABIE COMME OUIII CRITIOUE
}

\author{
PAR \\ Kal NIELSEN
}

\begin{abstract}
RÉSUMÉ : La notion de raisonnable est omniprésente dans l'œuvre de Rawls. Pourtant, plusieurs estiment que cette notion est problématique et qu'elle ne saurait avoir la portée critique dont Rawls l'investit. Dans cet article, je me propose de clarifier cette notion en soutenant que dans certaines de ses articulations conceptuelles, la notion de raisonnable peut effectivement jouer le rōle critique que lui assigne Rawls.

ABSTRACT: The appeal to reasonableness is ubiquitous in the work of John Rawls. Yet the concept itself is to be problematical and it is frequently tought that reasonability cannot have the critical weight that Rawls takes it to have. The concept is elucidated and, as well, some conceptualizations of reasonnability are articulated. It is argued that there are conceptualizations of reasonnability that can be critically deployed in the way Rawls presupposes.
\end{abstract}

\section{I}

Une lecture même superficielle de Political Liberalism, de "The Law of Peoples " et de "A Reply to Habermas " suffit pour constater que l'œuvre de Rawls contient de nombreuses références à la notion de raisonnable ${ }^{1}$, qui en est venue à jouer un rôle central dans la pensée de cet auteur. Or cet usage généreux du terme " raisonnable " n'a pas manqué de susciter des réactions critiques. On a souvent fait remarquer que la différence entre ce qui est raisonnable et ce qui ne l'est pas, voire le concept lui-mēme et les diverses conceptions du raisonnable sont trop vagues et trop liés au contexte pour supporter une évaluation critique. Certains, dont Wittgenstein, ont pensé que loin d'avoir un statut critique, le raisonnable pourrait bien ètre une manière de club idéologique dont sont exclus ceux qui ne pensent pas comme nous. L'usage de ce concept serait surtout d'ordre émotif (usage perlocutoire) ; à tout le moins, il serait à ce point dépendant

1. John Rawls, Political Liberalism, New York, Columbia University Press, 1993 ; "Reply to Habermas ". The Joumal of Philosophy. vol. 92, n 3. mars 1995, p. 132-180; "The Law of Peoples " dans Stephen Shute et Suzan Hurley (eds.), On Human Rights, New York, Basic Books, 1993, p. $42-82$. 
de la pratique particulière qu'il ne saurait avoir l'usage critique que Rawls lui reconnait ou tend à lui reconnaitre.

J'ai présenté ci-dessus le champ d'application auquel j'entends me consacrer. J'entends fournir maintenant quelques bonnes raisons de penser qu'en dépit des arguments qui s'y opposent, la notion de raisonnable est un véritable outil critique.

Comme on le sait, la dernière théorie de Rawls commence par une acceptation de l'idée que le pluralisme est un fait patent (la simple réalité sociologique) de nos sociètés modernes. On ne saurait passer à une société non pluraliste sans user de force autoritaire. Ce devrait être une société où la conception du prétendu bien commun est imposée d'une manière ou d'une autre à la majorité de ses membres, dans une alliance avec l'Église par exemple. On n'a qu'à évoquer l'Espagne de Franco, le Portugal de Salazar ou le Chili de Pinochet pour se rappeler qu'il s'agit d'une possibilité aussi réelle qu'inacceptable. Le pluralisme de Rawls sur la question des sociétés modernes doit donc être considéré à la lumière du réalisme sociologique plutôt que rejeté comme une extravagance idéologique.

Pour que l'appel de Rawls à une forme de consensus englobant ait un sens, le pluralisme de facto de nos sociétés capitalistes actuelles doit également pouvoir être dit raisonnable. En dépit de leurs rapports souvent conflictuels et de conceptions du bien qui peuvent ètre irréconciliables ${ }^{2}-$ Rawls suit Isaiah Berlin sur ce point -, les principaux groupes de la société constituent ensemble une forme de pluralisme raisonnable. Les membres des groupes raisonnables respectent ceux qui soutiennent des conceptions du bien différentes des leurs, car là où le pluralisme est raisonnable, on respecte les gens et leurs idées. Et s'il s'avère que les idées sont absolument déraisonnables, on continue de respecter ceux qui les soutiennent, même si on rejette leurs idées.

L'incompatibilité des différentes conceptions du bien ne facilite pas la tàche de développer des critères du raisonnable. Et mème si on pouvait trouver ou construire de tels critères, on n'aurait encore là que la condition nécessaire mais non suffisante pour qu'on puisse accorder que le consensus global joue bien le rôle que Rawls lui reconnait. Dès lors, qu'appelle-t-on "pluralisme raisonnable "? Guand pouvons-nous en jouir? Cette notion est-elle aussi objective et son objectivitê est-elle aussi applicable à la théorie politique que Rawls estime qu'elles le sont?

II

Stuart Hampshire, un critique de Rawls qui demeure sympathique à l'auteur, estime que celui-ci fait trop de cas de ce qui est raisonnable ou de ce qui ne l'est pas, négligeant les passions profondes qui forment le tissu de notre vie. Rawls oublierait

2. Concernant les voies de ce désaccord raisonnable, puisqu'on a affaire à des conceptions du bon au sujet desquelles les gens raisonnables ne parviennent pas à s'entendre, voir John Burt, " John Rawls and the Moral Vocation of Liberalism ", Raritan, 1995, p. 133-153. Il parvient à donner une interprétation sentie du libéralisme de Rawls sans tomber dans le vocabulaire énorme de cet auteur. 
également que les différences de socialisation internes à une culture entrainent des différences dans la conception des valeurs, concernant ce qui vaut la peine d'ètre fait et ce qu'il vaut la peine d'avoir, ces différences étant susceptibles de provoquer des conflits qui menacent la vie de toute société qui en est affligée. Suivant Hampshire, la position de Rawls se laisse ramener à une espèce de rationalisme déplacé $\dot{e}^{3}$. Cette conception qui prétend s'appuyer sur l'autorité finale de la raison serait " une illusion regrettable, remontant à la République de Platon, où Kant comme Rawls la puisent sans fausse honte ${ }^{4}$.

Nous aurions tendance à nous laisser impressionner par les discours sur la raison, surtout quand nous sommes philosophes : "Nous éprouvons toujours une sorte de crainte (achtung) lorsque les mots " raison" ou "irrationnel " nous sont lancés au visage dans un style kantien ${ }^{5}$ ". Si illusion il y a, elle est effectivement répandue chez les philosophes : on l'a déjà mentionné, Rawls utilise largement le vocabulaire rationnel, tout comme Habermas et Brian Barry le font par ailleurs.

Après avoir ètabli ce qu'il en est de la conception du "raisonnable ", qu'elle représente une forme de rationalisme, déplacé ou non, il reste à voir plus clairement ce que nous entendons lorsque nous disons que quelque chose est raisonnable.

\section{III}

Considérons, pour commencer, une conception du raisonnable qui soit dotée d'un contenu déterminé tout en échappant, dans la mesure du possible, à toute controverse. Cette caractérisation du raisonnable offre un contenu plus riche que les conceptions qui font appel uniquement à l'équité ou aux bons sentiments. Elle remonte à Socrate, et pourtant son contenu est encore solide; bien que compatible avec les idéaux libéraux, elle ne s'y laisse pas identifier et est donc parfaitement accessible aux non libéraux. Néanmoins, elle natteint pas à la pleine conception libéraliste de ce qui est raisonnable, celle-ci ayant, outre les caractéristiques générales, des traits qui lui sont propres.

La conception en question est présentée comme suit par McCarthy : être raisonnable, c'est avoir certaines vertus essentielles comme "l'ouverture d'esprit, l'absence de dogmatisme, une tendance à accepter les diffèrences, à écouter les autres, à considèrer sèrieusement leurs opinions et à modifier nos manières de penser, la capacité de voir les choses du point de vue des autres et celle de peser judicieusement le pour et le contre ${ }^{6}$. On n'a pas là une conception utopiste du raisonnable, mais une bonne description de ce que l'on doit faire pour s'approcher d'un comportement qui serait

3. Stuart Hampshire, Liberalism : The New Twist ", New York Review of Books, 12 aoūt 1993, p. 45.

4. Ibid.

5. Ibid.

6. Thomas McCarthy, "Kantian Constructivism and Reconstruction : Rawls and Habermas in Dialogue *, Ethics, octobre 1994, vol. 105, $n^{\circ} 1$, p. 62. 
complètement raisonnable (un idéal heuristique dont certaines personnes réussissent parfois à se rapprocher).

Certes, cette caractérisation des vertus qu'on associe au raisonnable comporte un certain flou, mais elle offre des applications précises. De nombreuses personnes pratiquent ces vertus de manière plus ou moins approximative, mais avec assez de régularité pour qu'on puisse les compter au nombre des personnes raisonnables, et il est évident qu'il y a aussi des gens qui ne le font pas. On a là un contraste évident.

De même, pour toute socièté donnée, à un moment donné, il existe un large consensus concernant les conceptions, croyances, attitudes et actions qui sont raisonnables et celles qui ne le sont pas. Bien qu'on puisse donner des explications qui s'appuient sur des particularités historiques et culturelles, il n'en demeure pas moins que dans tout l'Occident, tout au moins, les vertus mentionnées cidessus - qui sont des notions générales, mais non complètement indèterminées - ont été associées au raisonnable depuis très longtemps. McCarthy n'a pas tort lorsqu'il affirme que ces idées concernant ce qui est raisonnable, parfaitement évidentes aujourd'hui, sont aussi vieilles que Socrate. Cette conception de ce qui est raisonnable ne se rattache pas particulièrement au libéralisme et elle ne comporte rien qui la rende sujette à polémique.

\section{IV}

Considérons maintenant la conception libérale du raisonnable qui est largement celle de nos sociétés démocratiques capitalistes. Ses composantes ne sont pas l'apanage exclusif des sociétés libérales et peuvent apparaitre dans d'autres sociétés. Toutefois, l'ensemble de ses traits distinctifs tend à être caractéristique, voire particulier aux sociétés libérales.

Voici deux listes qui se rattachent à la conception libérale du raisonnable. La première comporte des éléments qui devraient apparaitre comme évidents; la deuxième est plus critique et pourrait susciter la controverse.

La caractérisation proposée est morale sans ètre moralisatrice, et Rawls sert ici de modèle. Il ne s'agit aucunement de fournir une conception non morale du raisonnable d'où on pourrait ensuite déduire la morale elle-mème ; on peut d'ailleurs douter qu'un tel projet soit intelligible. Il ne s'agit donc pas, comme Hobbes ou, de nos jours, David Gauthier, de partir d'une conception moralement neutre ou soidisant telle, pour en dériver la morale ou une de ses parties substantielles. Ce n'est pas là le rôle que Rawls entend faire jouer au raisonnable ; sa conception du raisonnable est morale et normative, politique, dès le départ, bien qu'on ne puisse la réduire à ces notions.

Les listes proposées plus bas sont longues et détaillées. On a d'abord une conception du raisonnable qui est, comme dirait Michael Walzer, large (thick) plutôt que minimale ${ }^{7}$. Sans trop me préoccuper

7. Sur cette large contextualité, voir Michael Walzer, Thick and Thin, NotreDame (Indiana), University of Notre-Dame Press, 1994. Voir aussi, du 
d'éliminer toute redondance, je donnerai une description permettant de distinguer le pluralisme raisonnable du pluralisme de facto. Il s'agira aussi de préciser ce qui est raisonnable par opposition à ce qui ne l'est pas, appliqué aux personnes et à leurs idées, croyances, attitudes, principes, plans, à la compréhension globale de ce qui est bien et à la conception de la justice qu'ont les citoyens vivant dans les sociétés libérales. De concert avec la conception de McCarthy, cette conception libèrale pourrait nous permettre d'évaluer le caractère raisonnable de sociètés entières, de manière telle qu'on puisse ensuite décider si l'ordre social proposé dans les circonstances de la modernité (qui sont celles où se passe notre vie) peut ou non ètre considéré comme supérieur à un ordre qui ne serait pas libéral.

\section{La conception libérale du ra is on nable}

Liste de traits sans controverse ${ }^{8}$

1. Les gens raisonnables doivent ètre prèts à modifier leurs idées, leurs croyances ou les actions qui en découlent, pour rendre possible une coopération sociale sur la base de la liberté et de l'égalité avec ceux qui pensent différemment ou qui ont d'autres croyances.

2. Pour leur propre bien, les personnes raisonnables "désirent vivre dans un monde social où elles pourront coopérer librement et en toute égalité avec les autres, sur une base acceptée par tous ${ }^{9}$ ". De façon caractéristique, ces personnes vont chercher à justifier leurs actions auprès des autres en s'appuyant sur des fondements qu'ils ne sauraient sensément rejeter ${ }^{10}$.

3. Les gens raisonnables désirent que dans le monde social, les relations entre les gens soient marquées par la liberté et l'égalité, entraînant une réciprocité dont chacun peut bénéficier.

4. Là oủ les gens sont raisonnables, certains travaillent à articuler et à présenter publiquement les fondements des relations sociales. Ces fondements devraient être tels que toute personne raisonnable les acceptera, une fois qu'elles les aura vus clairement. Les premiers cherchent à formuler les règles du monde social public, que tous endossent et à partir desquelles ils agissent ${ }^{\text {" }}$.

5. Toutes les formes de fanatisme sont rejetées.

6. Tous ceux qui sont tolérants manifestent leur tolérance et une ouverture d'esprit. L'intolérant est critiqué et son intolérance parfois réprimée : pourtant, mème l'intolérant peut ètre toléré, là où son intolérance ne fait aucun tort aux autres. Toutefois, il est évident que les personnes intolérantes ne sauraient ètre comptées au nombre des personnes raisonnables.

même auteur. Interpretation and Social Criticism, Cambridge. M. A.. Harvaud University Press, 1987

8. La liste comporte certainement des redondances. Plutōt que de chercher à produire une liste brève composèe d'énoncés canoniques, j'ai voulu, au moyen de différentes formulations ou nuances, saisir la conception libérale du raisonnable dans toute son " épaisseur *.

9. J. Rawls, Political Liberalism, p. 50.

10. Ibid., p. 49-50. Rawls suit ici Scanlon.

1 1. Ibid, p. 53. 
7. La tolérance dont il est question au point précédent s'accompagne du respect pour toutes les personnes.

8. Les personnes raisonnables ont un sens de l'équité, elles sont prètes à faire la moitié du chemin, à faire des compromis là où les questions de principe ne sont pas menacées. Ces personnes croient dans la valeur du respect mutuel et elles ont un sens de la civilité-12.

9. Les personnes raisonnables ont à cœur de régler les arrangements de manière à obtenir un équilibre entre les différents intérêts.

10. Les agents raisonnables veulent bien "écouter les autres "et ils sont "justes en modifiant leurs idées à propos ${ }^{13}$ ". Ces personnes s'engagent dans un dialogue public et une justification publique des idées.

11. Les accords doivent normalement ètre obtenus au moyen d'un dialogue où toutes les parties intéressées sont égales au point de vue de leurs dires comme de leurs intérêts. Les accords raisonnables doivent être obtenus de cette façon plutôt que par les armes de la rhétorique, les influences charismatiques, la force physique ou les marchandages où les plus forts sont sūrs de l'emporter sur les autres.

12. Chacun s'engage à ètre juste et équitable dans ses transactions avec les autres.

13. En cas de litige, on aura tendance à écouter toutes les parties concernées avant de décider d'une affaire pratique ou avant d'opter pour une certaine politique. On sera porté à réviser les jugements ou les politiques en vigueur à la lumière de nouveaux faits ou de nouvelles raisons. C'est dire que nous devons accepter comme évident que nos attitudes, nos manières de raisonner et nos styles sont faillibles de part en part.

14. On remarquera une tendance à l'autocritique de ses propres croyances, attitudes et manières de se comporter, aussi bien qu'une attitude critique, à la fois civile et prudemment sceptique, à l'endroit des institutions et des pratiques communes dans nos sociétés et dans les autres sociétés.

15. Dans les affaires publiques, on voudra n'avoir que des croyances qui soient en accord avec la réalité des faits reconnus. On cherchera à s'assurer de connaitre la majorité de tels faits ou en tout cas un nombre suffisant pour une connaissance pratique, sans éluder les faits à notre convenance.

16. À tout le moins dans les affaires publiques, on cherchera a) à fonder nos croyances sur l'évidence ; b) à ètre conséquent et cohérent; c) à énoncer le meilleur argument ou à rapporter le plus fidèlement possible la discussion et à accepter la force et les implications pratiques de l'argument ou de la discussion.

17. On cherchera à avoir des croyances et des politiques qui soient intelligibles et cohérentes à la lumière des circonstances dans lesquelles le raisonnement s'exerce normalement.

12. Ibid., p. 53-54.

13. Ibid., p. 123, 157, 163. 
18. On s'entend généralement pour penser que dans une société juste et bien ordonnée, aucune des parties impliquées dans un conflit ne saurait légitimement imposer ses convictions morales à l'ensemble de la société sans passer par les procédures habituelles et reconnues mais en usant de force.

19. Mème lorsqu'on ne réussit pas à s'entendre sur les points cruciaux, les résultats obtenus par des procédures correctes sont reconnus par tous les gens raisonnables.

\section{Liste sujette à controverse}

1. Les gens raisonnables reconnaissent que dans notre monde moderne complexe, il est inévitable que nombre de nos jugements les plus importants soient faits "dans des conditions où on ne saurait s'attendre à ce que des personnes consciencieuses et dotées de tous les pouvoirs que leur confère leur raison arrivent à la mème conclusion, même après une libre discussion ${ }^{14}$. Ce fait, qui relève de ce que Rawls appelle le poids du jugement, doit ètre pris au sérieux, ce qui implique qu'on ne cherchera pas à imposer à l'ensemble de la vie publique l'autorité de vues ou de plans qui sont contestés. Là où règne le dissensus, aucune des diverses croyances qui existent ne devrait ètre prise pour une norme gérant le comportement des gens en société.

2. "Une compréhension de la valeur n'est complètement raisonnable que dans le cas où ses défenseurs sont toujours disposés à la soutenir à mesure qu'ils reçoivent plus d'informations sur le sujet et les soumettent à leur réflexion ${ }^{15}$, "

3. Face à l'existence d'une diversité de conceptions du bien qui sont presque également raisonnables, nous devons nous restreindre, comme ètres raisonnables, à des exigences qui concernent l'ordre de la société. Là où on a, par exemple, des normes sociales qui sont similaires, il faut s'en tenir, lorsqu'il existe, au sol commun entre les diverses visions, en reconnaissant que la raison ne nous permet pas actuellement de trancher en faveur d'une seule vision morale et qu'en conséquence, nous devons éviter d'imposer à l'ensemble notre propre conception du bien commun. Seul ce qui est commun à toutes les conceptions du bien - ce qui fait l'objet d'un consensus parmi les personnes raisonnables - peut revendiquer l'autorité nécessaire pour fonder les institutions d'une société ou pour déterminer ce que l'on peut attendre des individus qui peuplent la société en question.

4. Les gens raisonnables reconnaissent qu'il est probable qu'il y aura toujours des gens qui soutiendront des conceptions déraisonnables ou irrationnelles. Il pourrait même s'agir là d'un truisme sur le plan empirique. Toutefois, pour les personnes raisonnables, "le fait que certaines personnes soutiennent des vues déraisonnables ne nous force pas à modifier notre conception de la justice de manière à la fonder sur un large consensus qui ferait appel à ces

14. Ibid., p. 217.

15. Ibid., p. 58. 
vues déraisonnables ${ }^{16}$ ". En d'autres termes, nous n'avons pas à nous accommoder de ce qui est déraisonnable. En effet, "si nous faisions une place à ce qui est déraisonnable dans la formulation des principes fondamentaux, nous ajusterions alors faussement nos principes sur le pouvoir de facto ${ }^{17}$. "

\section{$\checkmark$}

Il s'agit maintenant d'établir si on peut faire un usage critique de cette conception du raisonnable en l'appliquant à certaines croyances, certaines manières de faire ou de voir les choses. $\dot{A}$ moins qu'elle ne soit assez vague et indéterminée pour être compatible avec à peu près n'importe quoi ? Ou à moins qu'il ne s'agisse d'une conception idéologique ou ethnocentriste, une vue inclinée par le préjugé touchant la manière dont nous croyons que les choses devraient se passer? Loin de penser que l'idéologie libérale cherche à donner une facture rationnelle à une conception ethnocentriste, j'estime au contraire qu'elle fournit un instrument critique dans un contexte qui a toute l'objectivité et l'intersubjectivité historiques requises. Mais rien de plus. Cette conception est incapable de déterminer un point d'Archimède objectif non historique - une quête qui risquerait de n'avoir jamais de résultats substantiels.

Rawls appuie sa conception de la justice sociale sur la conception libérale du raisonnable. Il montre qu'elle est raisonnable au sens décrit plus haut, qui est plus fort que ce qui est proposé habituellement par un Brian Barry, par exemple, qui se trouve ètre un concurrent de Rawls. Celui-ci propose une conception qui est loin d'être simplement rhétorique et vide. Elle représente au contraire ce que les libéraux et ceux qui s'en rapprochent désirent voir se produire comme réalité politique dans nos sociétés encore loin d'être justes. Nous serions heureux de pouvoir constater que ce concept du raisonnable fonctionne dans nos sociétés.

Mais la question demeure de savoir si la conception libérale du raisonnable est capable de produire des normes sociales qui pourront prétendre être objectives. Dans un tel cas, le libéralisme aurait une valeur plus que simplement interne, quand les libéraux estiment que leurs vues sont meilleures que celles des non-libéraux, sans pouvoir les fonder autrement qu'en faisant appel à ce que les libéraux soutiennent et continueront de soutenir après müre réflexion. Á moins que tout ce qu'on puisse dire objectivement, c'est que les libéraux ont leur culture et leurs institutions, sans empêcher l'existence passée et actuelle de cultures différentes dotées de leurs propres institutions, qui peuvent rejeter des éléments importants de la conception libérale. Est-il possible d'êtablir qu'un ordre social est meilleur - ou plus raisonnable - qu'un autre ? Ou si notre conception du raisonnable est à ce point liée à une Weltanschauung donnée, qu'il est impossible de la déterminer plus objectivement?

16. Joshua Cohen, "Moral Pluralism and Political Consensus ", dans David Copp et al (eds.), The Idea of Democracy. Cambridge (England), Cambridge University Press, 1993, p. 282.

17. Ibid., p. 284. 
Finalement, notre conception du raisonnable est-elle autre chose qu'une manière de promouvoir le libéralisme?

Il est vrai que la conception du raisonnable qui est proposée ici est faite à la mesure du libéralisme : c'est l'idée qu'un libéral se fait de ce qui est raisonnable. Cette conception du raisonnable va de pair avec la conception du monde qui est celle des libéraux. Mais est-ce qu'elle nous donne pour autant les moyens d'établir ce qui est le plus raisonnable possible, que l'on soit libéral ou pas ? Peut-ètre que la chose la plus raisonnable ou la meilleure - mais certainement pas au sens libéral du terme "raisonnable "- serait de se faire Taliban musulman, Juif orthodoxe ou chrétien fondamentaliste comme Pat Robertson? Peut-on au contraire soutenir que toute personne vivant dans le monde moderne, si elle est bien informée, cherchera à être raisonnable de la manière décrite dans la conception libérale du raisonnable, sans méme avoir besoin d'être libérale? Dans le meilleur des cas, nous aurions uniquement établi ce qui suit : si nous voulons ètre des personnes libérales ou si nous sommes favorables à ceux qui le sont, nous voudrons que cette libéralité se manifeste dans notre conception du raisonnable. Or, dans le cas contraire, rien ne prouve que pour être raisonnables sans plus, nous devions être libéraux, c'est-à-dire raisonnables au sens de la conception libérale. Mais alors les gens se divisent-ils simplement en ceux dont la sociabilité les conduit au libéralisme et ceux qui n'empruntent pas cette voie du libéralisme?

On n'a pas besoin de se réduire à l'idéologie libérale du raisonnable. On peut faire appel à des arguments autorisant le passage de la conception générale du raisonnable (qui remonte à Socrate) à la conception libérale proprement dite, en s'appuyant sur des croyances considérées comme plausibles ou mème largement rëpandues, bien au-delà de l'ethos libéral, sur des évidences morales qui ne dépendent pas de la conception libérale du raisonnable. Ainsi agencées, les deux conceptions engendreront des normes solides pouvant ètre soumises à une large critique sociale.

Prenons un exemple. Les conceptions fondamentalistes, qu'elles soient juives, chrétiennes ou musulmanes, ne sont pas jugées raisonnables, même si on s'en tient à la conception la plus générale, culturellement parlant - celle qui a èté formulée d'abord par Socrate sans parler de la conception proprement libérale du raisonnable. Suivant la conception générale, d'abord, les gens raisonnables sont ouverts d'esprit ; ils sont prêts à envisager sérieusement les opinions d'autrui ; ils cherchent à éviter tout dogmatisme; ils acceptent de parler des différences; ils cherchent à voir les choses non seulement à partir de leur point de vue mais également à partir de celui des autres ; dans les cas litigieux, ils sont capables de peser le pour et le contre. Or, les fondamentalistes ne font rien de cela. Dieu leur a parlé et Il leur a révélé la Vérité Ultime sur la Vie, la manière dont les gens doivent vivre et l'ordre du monde. Ils ont des écrits sacrés qui leur montrent la Voie. Ils sont certains que c'est bien Dieu qui s'adresse à eux, ils savent ce qu'Il pense au sujet de telle ou telle chose et plus généralement, au sujet de la manière dont les gens doivent vivre. Les Ecritures livrent une vérité infaillible : ceux qui ne croient pas cela sont perdus dans l'illusion et corrompus par le péché. On ne doit 
donc pas les ècouter. Certains fondamentalistes iront jusqu'à l'anathème. Bref, les fondamentalistes croient que la vérité ultime au sujet de la vie leur a été révélée par Dieu lui-même, dont ils sont les enfants.

Une telle conception est déraisonnable. Elle s'oppose à la signification la plus simple du raisonnable comme à la plus sophistiquée. Évidemment, les fondamentalistes ne pensent pas que leurs conceptions sont dëraisonnables ou irrationnelles (c'est peut-ètre là quelque chose que personne-y compris les personnages de Dostoïevski - ne dirait de lui-mēme sans ironie ou sans autre explication). Mais les actions et les croyances exprimées par les fondamentalistes montrent que leurs conceptions sont déraisonnables lorsqu'on les mesure à l'aune de la signification du terme "raisonnable " présentée plus haut. Ceci reste vrai mème si on se limite à la conception générale remontant à Socrate.

L'engagement des fondamentalistes radicaux qui sont des adultes vivant dans une culture moderne qui leur offre d'autres choix est déraisonnable et les conceptions qu'ils soutiennent le sont également. Ils sont purement et simplement déraisonnables. Encore une fois, on n'a pas besoin d'entendre le terme "raisonnable "suivant la vision de l'idéologie libérale ; il suffit de prendre la signification générale, socratique, qui trace les limites du jeu de langage que nous jouons avec le mot "raisonnable". Au cas où quelqu'un trouverait que j'ai dépeint les fondamentalistes comme pires qu'ils ne sont en réalité, je dirais la même chose de quiconque soutenant les vues que j'aurais ici attribuées faussement aux fondamentalistes. Ces vues nous donnent une idée claire de ce qui est déraisonnable. Même si, contrairement à ce que je pense, cette conception n'est pas aussi fréquente que je le crois, il y a des exemples et c'est de ceux-ci dont il s'agit. Laissons donc aux sociologues le soin de décider si la caractérisation des fondamentalistes qui a été donnée plus haut est correcte.

Par ailleurs, une des raisons pour lesquelles nous n'aimons pas dire qu'une personne est déraisonnable ou qu'à tout le moins certaines de ses conceptions le sont est qu'il s'agit là d'une critique très sévère. Le simple devoir de civilité souligné par Rawls milite contre ce genre de critique. Un libéral pourrait se demander s'il n'est pas déraisonnable d'adresser cette critique à quelqu'un. Est-ce que cette critique ne tombe pas elle-même dans une forme de fanatisme? Il faut donc recommander l'exercice de la prudence et de la charité, mais mème ces vertus ont leurs limites. Lorsque quelqu'un affirme qu'il a La Vérité, qu'il ne veut pas entendre ce que les autres pourraient avoir à dire parce qu'il se place au-delà de toute controverse (dans les bras de Dieu), alors cette personne est déraisonnable et il faut le dire, avec civilité et politesse sans doute, mais surtout avec fermeté.

La conception libérale du raisonnable que je défends, de concert avec les John Rawls, Brian Barry et Joshua Cohen, n'a pas besoin de s'accommoder du déraisonnable. Nous n'avons pas à ajuster notre conception de la justice ou nos principes à l'existence de personnes et de conceptions déraisonnables. En résistant au pouvoir de ces personnes ou de ces conceptions déraisonnables, nous ne sommes pas intolérants ou fanatiques (en opposant un pouvoir à un autre). 
mais nous résistons à ceux qui veulent nous imposer leurs vues sans passer par la raison. Nous restons ouverts à leurs arguments, nous voulons bien les écouter présenter leur cas, mais ils doivent le faire en recourant à ce qui peut ètre défendu par les armes de la raison. On ne saurait simplement leur céder lorsqu'ils affirment qu'ils sont dans leur droit et que ce qu'ils disent doit ètre fait ou accepté. Ce n'est pas nous qui sommes fanatiques en leur résistant, mais eux qui le sont lorsqu'ils soutiennent des vues déraisonnables et insistent pour les imposer.

Cette discussion au sujet du fondamentalisme montre que notre conception du raisonnable peut servir d'outil critique d'une façon qui n'a rien d'idéologique : elle sert à critiquer les visions fondamentalistes extrêmes en fournissant des arguments pour les rejeter. Cette critique peut être étendue à quelqu'un qui prétend, comme Alvin Plantinga le fait à la suite de John Calvin, avoir une appréhension directe de Dieu qui se situe au-delà de toute controverse $e^{18}$. Toutefois, l'application du concept de raisonnable comme outil critique n'est pas toujours aussi claire.

Ainsi, comme Rawls et Barry, j'ai une conception égalitariste de la justice. Toutefois, celle-ci ne saurait ètre fondée uniquement sur ce qui est raisonnable. En effet, certaines sociétés passées et actuelles ont des théories aristocratiques ou hiérarchiques de la justice qui ne doivent rien aux conceptions libérales. Ces sociétés ont également leurs philosophes et autres penseurs qui articulent les conceptions en vigueur chez eux. Or, ces penseurs peuvent parfois ètre considérés comme raisonnables suivant la conception générale de ce qui est raisonnable. Ils peuvent ètre et sont parfois accessibles, non fanatiques, non dogmatiques, ouverts à l'examen des évidences, à la discussion des différences et même capables de voir les choses du point de vue des autres et de peser le pour et le contre dans des situations données. Ils peuvent donc ètre considérés comme raisonnables de mème que leurs conceptions, ce qui n'empēche pas cellesci de favoriser une hiérarchie sociale plutôt que le libéralisme.

Ces personnes pensent qu'une vision réaliste et non sentimentale de la nature humaine nous mène tout droit à la conclusion que tout le monde n'a pas les mèmes capacités morales et intellectuelles : tous les gens ne sont pas également autonomes, capables d'agir avec réflexion et de vivre intelligemment, sans parler de participer à la vie publique ou de comprendre comment une société doit ètre ordonnée pour bien fonctionner. Voilà pourquoi le pouvoir doit être réservé à une classe de gens supérieurs, qui sont seuls à pouvoir produire une société où les principes de respect et de justice seront sauvegardés. Gardant à l'esprit la maxime de Nietszche qui dit que la fin de la justice est de traiter également ce qui est inégal, ces personnes supérieures se placent au-dessus de la masse des gens inférieurs.

18. Alvin Plantinga approuve John Calvin lorsque celui-ci affirme qu'instinctivement, chaque être humain est capable d'appréhender la đivinité. C'est là une possibilité qu'il place " au-delà de toute controverse *. Voir Alvin Plantinga, "Belief in God ", dans Michael Boylan (ed.), Perspectives in Philosophy. Forth Worth (Texas), Harcourt Brace Jovanovich, 1993, p. 405. De John Calvin, voir Institutes of the Christian Religion, Philadelphia, Westminster Press, 1960, livre I, chap. 3, p. 43-44. 
Depuis leur position privilégiée, ils déterminent la structure de l'ordre social en attribuant à chacun la position qui convient à son mérite. Dans leur société, certains individus sont subordonnés à d'autres. De nombreux sujets - incluant des questions morales, politiques et religieuses - ne sauraient ètre discutés ou mème compris correctement par la vaste majorité de la population, qui doit donc accepter les dires de ses dirigeants. Or, on peut soutenir une telle conception aristocratique - qui est très distante de notre conception libérale - en usant de tolérance, civilité, douceur, sans oublier une bonne dose de paternalisme. Après tout, tous les conservateurs ne sont pas des moralistes tonitruants.

Certains conservateurs passent le test de la conception générale du raisonnable. Il reste qu'ils ne sont pas raisonnables au sens de la conception libérale plus large que nous avons tenté de dégager. Toutefois, les parties de la conception libérale large qui nous permettent de douter que ces personnes conservatrices soient tout à fait raisonnables sont sujettes à controverse. Le sens du raisonnable peut ètre défini de manière persuasive, voire il peut fonctionner comme un instrument idéologique aux mains du libéralisme, de la modernité et de la post-modernité. Il semble alors avoir peu de choses en commun avec un outil critique utilisè pour questionner la société.

La conception libérale tient pour acquis que les gens raisonnables désirent vivre dans un monde social où les relations entre les individus sont marquées au sceau de la liberté et de l'égalité. C'est là ce que les libéraux doivent garder à l'esprit lorsqu'ils cherchent à déterminer l'ordre social. Si nous voulons ètre libéraux au sens de la conception libérale, nous devons reconnaître qu'au point de vue moral, tous les êtres humains sont égaux, et cette acceptation de l'égalité doit se refléter dans nos politiques. Les personnes raisonnables au sens du libéralisme croient que la vie de tous et chacun est d'égale valeur: c'est là une opinion partagée par tous les libéraux, qu'ils soient libéralistes, démocrates, socialistes ou communistes, qu'ils soient de droite comme Robert Nozick et David Gauthier ou de gauche comme G. A. Cohen et Jon Elster. Mais cette opinion n'est pas partagée par les aristocrates opposés au libéralisme, qui estiment que cet élément de la conception libérale et communiste du raisonnable relève du sentimentalisme et du moralisme (encore un mot de Nietzschel plutôt que de la raison. Pour les conservateurs, ce sont des sentiments et des sympathies libėrales et communistes qui portent la volonté des libéraux de vivre dans un monde où tous les hommes sont libres et égaux. Les libéraux sont victimes d'une illusion s'ils croient que le raisonnable requiert l'engagement égalitaire. Personnellement, j'estime que l'égalitarisme est consistant avec le raisonnable sans être pour autant absolument requis par ce concept.

Les conceptions morales ou normatives, incluant les appels à la justice sociale ou politique, ne sauraient être dérivées de la seule conception générale du raisonnable, ou de cette conception et d'une bonne connaissance des faits qui n'ont rien à voir avec la morale. À mon avis, non seulement il est impossible de dériver la morale du raisonnable, mais on ne saurait pas plus la dériver de la rationalité. Hobbes, et plus récemment David Gauthier, ont cherchè à prouver la 
rationalité de la moralité en partant d'une rationalité pratique pure. Or, tout ce qu'on peut prouver, c'est qu'une certaine sorte d'égoïsme éthique est exclue, ce qui laisse ouvert le champ des diverses visions morales. La faussetê et l'incohérence de l'égoïsme éthique ne font pas de l'égoïsme personnel une attitude irrationnelle dans toutes les circonstances : ainsi, le fou de Hobbes pratique une forme d'amoralisme prudent ${ }^{19}$.

Le concept de raisonnable, distinct de la rationalité en ce qu'il a quelque chose à voir avec la morale, paraît mieux placé pour fonder la moralité que la rationalité. Mais comme on l'a déjà vu, des conceptions également raisonnables comme le sont la conception hiérarchique de la justice et la conception libérale égalitaire sont pourtant en conflit. On ne saurait, à partir des seules ressources du concept du raisonnable, justifier que l'on opte pour un ordre social libéral plutôt que hiérarchique. Pour ce faire, il faut dépasser la conception socratique du raisonnable et prendre en compte les choix moraux et politiques de la conception libérale. On ne saurait justifier l'égalitarisme ou une notion plus faible d'égalité morale (à la Nozick) sans inclure dans le concept de raisonnable la conception libérale large. Mais en injectant une notion égalitaire dans notre concept du raisonnable, nous avons défini le mot raisonnable de telle manière que des personnes qui sont raisonnables de façon évidente ne peuvent plus ètre dites raisonnables.

Mon but n'est pas d'opposer raison et passion, ou de ruiner la distinction en donnant la préséance à l'une plutôt qu'à l'autre. Je préfère parler d'un vaste équilibre réflexif dans lequel les deux notions ont un rôle à jouer : il est important de tenir compte à la fois de ce qu'il est raisonnable de croire et de faire, et de nos sympathies les plus réfléchies. Pour produire une conception de la justice et plus généralement de la morale qui soit équilibrée, nous devons savoir ce qui arrive (il s'agit de mettre nos points de vue en accord avec la réalité), considérer judicieusement les différentes raisons qui militent en faveur d'un ordre plutót que d'un autre, et nous devons aussi clarifier pleinement nos sentiments à l'endroit des différentes manières d'organiser la vie sociale. Il s'agit de rassembler ces différents facteurs - et d'autres encore - dans une conception cohérente.

L'équilibre réflexif auquel nous pensons est une forme de justification pratique. Les jugements pouvant faire partie de ce large équilibre sont ceux qui sont considérés comme justifiés à ce momentlà, des jugements seulement pour un temps. Ce que nous considérons comme juste, bon, rationnel ou raisonnable fait partie d'un vaste équilibre qui peut constamment ètre remis en question. Toutefois, les jugements dont nous disposons nous permettent de forger une conception cohérente de ce qu'il est justifié de faire et de croire. Le concept de raisonnable est central, mais il faut aussi compter avec les sympathies et les sensibilités humaines, sans qu'aucun des différents facteurs puisse lemporter sur les autres. Rien ne mérite

19. Voir Kai Nielsen, Why be moral ?, Buffalo, New York, Prometheus Books, 1989 , p. 143-206. Voir également Debra A. DeBruin, "Can One Justify Morality to Fooles ? ", Canadian Joumal of Philosophy, vol. 25, $n^{\circ} 1$, mars 1995, p. $1-32$. 
d'ètre appelé le fondement réel de la moralité : ni la raison, ni les sentiments, ni les faits, ni l'intuition, ni les intérêts. Sans être arbitraire ou conventionnelle, la moralité n'a pas de vêritable fondement. Tout ce qui existe, c'est la conception holiste d'un vaste équilibre réflexif.

C'est Rawls qui a remarqué que "le critère général du raisonnable est un vaste équilibre réflexif ${ }^{20}$ ". Il n'existe pas une seule chose dont on puisse dire qu'elle fonde toute la morale ou même la partie de la morale qui ne serait pas complètement conventionnelle. Avec Stuart Hampshire, dont le holisme est comparable à celui de Rawls ou de guine, on peut affirmer que "la philosophie morale [...] consiste à chercher quels sont les fondements raisonnables de la moralite ${ }^{21}$ ". Venant d'un philosophe qui estime que Rawls succombe à une forme de rationalisme erroné, c'est dire que la philosophie morale doit établir le caractère rationnel de nos conceptions morales, montrant qu'elles ne sont pas un simple fourre-tout, ce qui se fait en montrant comment nos conceptions morales font partie d'un ensemble cohèrent avec le reste de ce que nous croyons, sentons ou savons comme ètres raisonnables. Les considèrations relatives à la raison sont cruciales, mais il ne s'agit pas de dire que la raison est le fond du fond, car ce fond absolu n'existe pas : c'est là uniquement une expression rhétorique ou idéologique. La conception holiste et cohérentiste proposée

20. J. Rawls, "Reply to Habermas ", p. 141.

21. Stuart Hampshire, "A New Philosophy for the Just Society ", The New York Review of Books, vol. 18, $n^{\circ} 3,24$ fév. 1972, p. 39. 
n'est pas non plus ethnocentriste, mais elle fait partie de nos outils critiques, dont l'usage doit s'accompagner d'une reconnaissance de l'importance de nos sympathies morales, sans faire de la raison l'esclave de nos passions ${ }^{22}$.

Département de philosophie

Université de Concordia

Traduit de l'anglais par Josette Lanteigne

22. Une première version de cet article fut présentée lors du symposium sur Rawls organisé par la Société de Philosophie du Québec, en 1995. Á cette occasion, Michel Seymour s'est demandé si le holisme rattaché à la notion d'équilibre réflexif était sémantique, épistémologique ou méthodologique. Comme il s'agit d'une méthode pour fixer la croyance, j'ai d'abord cru bon de répondre qu'il devait s'agir d'une forme de holisme méthodologique. Mais il faut reconnaitre que les avocats de l'équilibre réflexif n'emploient pas ce genre de distinction, qui dans l'ensemble cause plus de mal que de bien. Par ailleurs, on pourrait soutenir que la position holiste a des résonances qui sont à la fois méthodologiques, épistémologiques et sémantiques. Notre holisme pourrait ètre dit sẻmantique, puisqu'il s'intéresse aux concepts et aux conceptions de justice, bonté, raisonnable, garantie, justification. explication, personne, morale, politique, etc. Prenons les concepts de justice, de bonté et de raisonnable. Pour Rawls, Norman Daniel ou moi-même, il s'agit d'expliquer et de justifier une certaine conception politique de la justice et les croyances morales et politiques qui lui sont reliées, d'agencer ces différents éléments de manière à produire un tout cohérent, qui s'accorde avec le reste de nos croyances et convictions, nos sympathies, antipathies, etc. Voilà qui implique la sémantique et l'épistémologie, bien que les sémanticiens et les êpistémologues pourraient ne pas approuver notre manière de décrire les choses. Comme il s'agit d'une méthode pour fixer la croyance. on peut parler de holisme méthodologique ou épistêmologique. La meilleure chose à faire, cependant, est encore d'éviter ces catégories, qui n'ont pas grand-chose à voir avec ce qu'on entend par holisme. Lorsque l'on cherche à articuler une conception de la justice, il n'est pas nécessaire d'opter pour une position atomiste ou moléculaire. Pour acquérir une bonne compréhension des phénomènes, il s'agit plutôt de mettre au point des modèles généraux pour la croyance, la conviction et la conception, que nous voudrons ensuite rassembler dans un tout cohérent où tous les élèments sont reliés entre eux et présentés dans leurs relations les uns avec les autres. Voir mon article * How to Proceed in Social Philosophy : Contextualist Justice and Wide Reflective Equilibrium ", Queen's Law Journal, vol. 20, $\mathrm{n}^{\circ} 1$, automne 1994, p. 89-138; idem, After the Demise of the Tradition, Boulder (Colorado), Westminster Press, 1991, p. 195-248; idem. "Relativism and Wide Reflective Equilibrim ", The Monist, vol. 76, $\mathrm{n}^{\circ} 3$, juillet 1993, p. 316-332. 